\title{
Comparison of detecting Legionella Pneumophilla with urinary antigen test in teaching hospitals
}

\author{
Mohammad Amin Fazeli ${ }^{\mathrm{a}}$, Mohammad Javad Mohammadi ${ }^{\mathrm{b}, \mathrm{c}}$, Seyed Mojtaba Mousavian ${ }^{\mathrm{d}}$, \\ Rohangiz Nashibi ${ }^{\mathrm{e}}$, Seyed Mohammad Alavi ${ }^{\mathrm{e}, *}$ \\ a Infectious Diseases Ward, Razi Hospital, Ahvaz Jundishapur University of Medical Sciences, Ahvaz, Iran \\ ${ }^{\mathrm{b}}$ Asadabad School of Medical Sciences, Asadabad, Iran \\ ${ }^{\mathrm{c}}$ Department of Environmental Health Engineering, School of Public Health AND Environmental Technologies Research Center, Ahvaz Jundishapur University of Medical \\ Sciences, Ahvaz, Iran \\ ${ }^{\mathrm{d}}$ Department of Microbiology, Faculty of Medicine, Ahvaz Jundishapur University of Medical Sciences, Ahvaz, Iran \\ ${ }^{\mathrm{e}}$ Health Research Institute, Infectious and Tropical Diseases Research Center, Ahvaz Jundishapur University of Medical Sciences, Ahvaz, Iran
}

\section{A R T I C L E I N F O}

\section{Keywords:}

Legionella pneumophilla

Antigen test

Patients

Teaching hospitals

\begin{abstract}
A B S T R A C T
Introduction: Pneumonia is one of the most common causes of mortality among respiratory diseases, Legionella pneumophila (L.pneumophila) is an important cause of pneumonia in the world. Despite the evidences of favorable epidemiological conditions and the presence of the pathogen in Ahvaz, this disease has not been investigated properly.

Objective: The aim of this research project was to determine the frequency of L.pneumophila with urinary antigen test in hospitalized patients with Community-Acquired Pneumonia (CAP) in need and non-need intensive care in Ahvaz.

Materials and methods: In this case-control study, two groups of 70 patients with CAP need Intensive-Care Unit (ICU) as cases, and non-need ICU as controls who were hospitalized in teaching hospitals of Ahvaz were selected and tested for urinary antigen by a commercial kit (Legionella Kit, 2016, Belgium). Data were analyzed using SPSS 15 software by descriptive statistics.

Results: According to the data analysis, 140 patients, 5 patients (3.6\%) were positive for the Legionella urinary antigen, $3(4.3 \%)$ were in case and $2(2.9 \%)$ in control group. Although cases in compare with controls were more likely to be smokers, having serum sodium less than 130 (meq/lit) and less than 1500(cell/ml) lymphocytes in cell blood count, but statistically were not significant. In the region of study, L.pneumophila can cause respiratory infection but is an uncommon cause of pneumonia in hospitalized patients.

Conclusion: This disease primarily involves smokers and people with immunodeficiency; especially those with low lymphocyte early in admission. Therefore, in patients with a history of smoking and severe pneumonia requiring hospitalization in ICU, Legionella should be kept in mind.
\end{abstract}

\section{Introduction}

Pneumonia is one of the most common causes of death among infectious diseases. ${ }^{1}$ One of the pathological agents of severe forms of the disease is L.pneumophila. ${ }^{2}$ A flood of small, gram-negative, obligate aerobic, it is worth noting that so far about 50 species, 20 species of Legionella have been identified that are pathogenic for humans. Among the most common species are $\mathrm{p}$ L.pneumophila. ${ }^{3}$ Air-conditioning, cooling towers, indoor swimming pools, water coolers and piping hot water are the most cause these microorganisms. ${ }^{4,5}$ These microorganisms can cause community-acquired pneumonia or hospital forms and is sporadic or epidemic. ${ }^{3-6}$ L.pneumophila in some studies is the fourth most common cause of pneumonia are frequently severe and appear atypical. ${ }^{4-7}$ If proper diagnosis, appropriate treatment to reduce mortality, reduce costs and rational use of antibiotics and prevent the outbreak will be possible. ${ }^{6}$ There are several risk factors in these patients. In some studies, age, smoking, heart disease, chronic pulmonary disease, diabetes mellitus, kidney failure, organ transplantation, immunodeficiency, some types of cancer and age over 50 years were risk factors. Identifying risk factors can help people to take precautionary

\footnotetext{
* Corresponding author. Health Research Institute, Infectious and Tropical Diseases Research Center, Ahvaz Jundishapur University of Medical Sciences, Iran. Tel.: + 09161184916; fax: +986133387724.

E-mail addresses: aseyedmohammad119@yahoo.com, alavi-sm@ajums.ac.ir (S.M. Alavi).
} 
measures. $^{1}$

According to the conditions for the presence and survival of the micro-organisms in the province and microbiological survey as well as research in 2007-2008 in Ahvaz hospitalized patients with a diagnosis of pneumonia and determine the seroprevalence of $17 \%$ for L.pneumophila, bring in the presence as an important etiologic agent of pneumonia in the province, looks serious. ${ }^{4,8}$

Sputum culture, urinary antigen test and serologic there are different methods of for identify L.pneumophila. ${ }^{4,6-10}$ Legionella urinary antigen test is recommended in patients with severe pneumonia, which in some studies, the sensitivity and specificity is $95-100 \% .^{4,6-10}$

Extensive research on the world level in terms of diagnostic and therapeutic methods that include sporadic and epidemic L.pneumophila done, and in some countries annual reports and statistics presented, the major diagnostic procedure in most centers is urinary antigen test. ${ }^{11-13}$ Research in this area is limited to a case in our country, for comparing Legionella pneumophila urinary antigen detection by sputum culture in Tehran that urinary antigen test results have been better than sputum culture. ${ }^{14}$ L.pneumophila studies based on urinary antigen tests in Khuzestan province has not been conducted. According to what was said, it is necessary to investigate the presence of L.pneumophila urinary antigen in patients with pneumonia in the country and in particular in the province.

The objective of this study was to determine the presence of L.pneumophila urinary antigen and its risk factors in patients with severe pneumonia requiring hospitalization in ICU and those don't need ICU. It is hoped that it will be useful to provide better services to patients with severe pneumonia.

\section{Materials and Methods}

\subsection{Study design}

This prospective study was conducted to in Ahvaz city, Iran. Cases were chosen from teaching hospitals of Ahvaz from February 2015 to February 2016. Ahvaz is the capital of Khuzestan province in the southwest of Iran and it is located in the Middle East. ${ }^{15,16}$

The survey was conducted in Ahvaz teaching hospitals, admitted patients over 18 years of age and with primary diagnosis of pneumonia enrolled the study. It was the first complete history of the patient and disease characteristics including age, gender, location, occupation, comorbidity (diabetes, cancer, Human immunodeficiency virus (HIV), taking $20 \mathrm{mg}$ of prednisone or other steroids equivalent for more than a month, liver and kidney disease), smoking history, recent travel, use of antibiotics in the last month, recent hospitalization, symptoms (including fever, cough, shortness of breath, chest pain, purulent sputum), were recorded. Patients were examined in full and vital signs including blood pressure, respiratory rate, pulse rate and body temperature through the patient's mouth in minutes as well as normal or abnormal lung examination (including changes in breath sounds, rhonchi, crackles, wheezing) was recorded. Also, patients were taken with blood samples for Cell Blood Count (CBC), Blood Urea Nitrogen (BUN), K, Na, Albumin, Atrial Blood Gas (ABG) and chest radiography. Patients who have new radiographic pulmonary infiltration with at least one of the fever (temperature above $38^{\circ}$ ), cough, purulent sputum, pleuretic chest pain, leukocytosis (White Blood Cell $(W B C)>12000)$ or leukopenia (WBC < 4000), were regarded as pneumonia. Patients diagnosed as pneumonia and given the history, clinical signs, tests and criteria according to Infectious Diseases Society of America (IDSA) were divided in two groups; in need of ICU care as ICU group (cases) and non-ICU group (controls). IDSA criteria to need care in the ICU include intubation in the initial hospitalization, and shock needing vasopressor or having 3 or more of the following criteria; 1, BUN $>20 \mathrm{mg} / \mathrm{dl}, 2$. $\mathrm{RR} \geq 30 / \mathrm{min} ; 3$, Multilobar lung infiltration on radiography; $4, \mathrm{PaO}_{2}$ / $\mathrm{FiO}_{2}<250$; 5, Platelet count $<100000 / \mathrm{ml} ; 6$, (Oral) T $>38 \mathrm{C}$; 7, WBC (cell/ml) < 4000; 8, PH < 7.30-7.35; 9, Lab < 3.5(gr/dl); 10,WBC
$($ cell/ml) $>20000 ; 11, \mathrm{HR}>120 \mathrm{~min} ; 12, \mathrm{Na}<130 \mathrm{meq} / \mathrm{lit}$ and 13 , Age $>80$ (years).

Including criteria were as: acute phase of the disease in less than $72 \mathrm{~h}$ had passed from the start antibiotics, Ahvaz residents during the last month before and did not travel, not hospitalized due to other diseases (heart failure, asthma, etc.) and no history of hospitalization during the 3 months ago. Controls were selected as cases but didn't need ICU care.

To the patients or their families about the benefits of urinary antigen test explained, after obtaining permission from $5 \mathrm{cc}$ of their urine samples in the test tube collected and was sent to the laboratory as soon as possible, urine samples, centrifuged and was condensed and then (Legionella kit, Belgium, 2016) used according to guidelines and brochure sets, experiments were conducted and the results were positive, negative or inappropriate recorded if inappropriateness, was repeated. By taking samples in previous studies and considering the possibility of scholars to increase the sample size was 70 people per group. Descriptive statistics and graphical data used for better description, independent $t$-test and chi-square tests were used to analyze the data.

\subsection{Statistical methods of analysis}

Descriptive statistics was used and for qualitative variables. Data were analyzed using SPSS 15 software.

\section{Result}

In this study, 140 patients were hospitalized as pneumonia81 ! (57.9\%) men and $59(42.1 \%)$ were women, mean age $51.44 \pm 18.2$ years. 77 patients (55\%) living in the city and 63 patients (45\%) were from rural areas, $26(18.6 \%)$ were smokers, 12 (8.6\%) had diabetes mellitus. Urinary antigen in $5(3.6 \%)$ were positive. The mean white blood cells in patients was $10113 \pm 6999(\mathrm{cell} / \mathrm{ml})$ and lymphocytes average $1797 \pm 865$ (cell/ml), respectively. In 15 lymphocytes were less than $1000(\mathrm{cell} / \mathrm{ml})$. The mean serum sodium in patients was $133 \pm 6 / 9$ (meq/lit). The control group included 70 patients with community acquired pneumonia not requiring intensive care and the case group consisted of 70 patients with severe pneumonia requiring intensive care. Comparison of demographic characteristics, smoking and diabetes mellitus between the two groups are shown in Table 1. Comparison of experimental results for lymphocytes, leukocytes, sodium and legionella urinary antigen between the two groups are shown in Table 2.

In men of non-ICU group, 2 patients (5\%) and in women, no cases of Legionella were found. In men of ICU group $2(4.9 \%)$ and in women 1 (3.4\%) tested positive for Legionella were found. Of patients in non-ICU group living in city, $1(2.6 \%)$ and in rural area $1(3.1 \%)$ tested positive for Legionella were found. Of patients of ICU group in the city, 2 (5.1\%) and in rural area $1(3.2 \%)$ antigen was positive for Legionella. Controls smokers had no case of Legionella and of nonsmokers 2 (3.5\%) were positive for Legionella urinary antigen test. Of Smokers required intensive care 2 cases $(15.4 \%)$ were urinary antigen positive and in nonsmokers $1(1.8 \%)$ was positive for Legionella urinary antigen. In non-ICU patients with diabetes Legionella urinary antigen was not found to be positive, but in non-diabetics $2(3.1 \%)$ were positive. In ICU group, diabetic patients, $1(14.3 \%)$ and in non-diabetics 2 patients

Table 1

Compares demographic characteristics between the two groups.

\begin{tabular}{llll}
\hline Variable & Case & control & P-value \\
\hline Age (mean \pm SD) & $51.6 \pm 18$ & $51.3 \pm 18.2$ & 0.91 \\
Gender (male) & $41(58.5 \%)$ & $40(57.1 \%)$ & 0.78 \\
Location (city) & $39(55 \%)$ & $38(54 \%)$ & 0.78 \\
Smoking & $13(18.6 \%)$ & $13(18.6 \%)$ & 0.99 \\
Diabetes & $7(10 \%)$ & $5(7.1 \%)$ & 0.55 \\
\hline
\end{tabular}


Table 2

Comparison of experimental results between the two groups.

\begin{tabular}{llll}
\hline Variable & Case & Control & P-value \\
\hline Lymphocytes(cell/ml) (mean $\pm \mathrm{SD})$ & $1734 \pm 865$ & $1860 \pm 0.865$ & 0.39 \\
Leukocytes (cell/ml) (mean $\pm \mathrm{SD})$ & $10236 \pm 6669$ & $9989 \pm 6669$ & 0.83 \\
Sodium (meq/lit) (mean $\pm \mathrm{SD})$ & $133 \pm 6.97$ & $134.7 \pm 6.97$ & 0.81 \\
Legionella urinary antigen positive & $3(4.3 \%)$ & $2(2.9 \%)$ & 0.99 \\
\hline
\end{tabular}

(3.2\%), urine test was positive for Legionella. Intensive care needed patients who had low sodium, 1 of whom (6.3\%) and in those with higher sodium of 130 (meq/lit) one of them (1.9\%) were positive for Legionella urine test. Of patients who need intensive care that sodium less than 130 (meq/lit) one of them (5.3\%) and in those who have had sodium above 130, 2 of them (3.9\%) were positive Legionella urinary test. In non-ICU patients and lymphocytes count higher than 1500 (cell/ $\mathrm{ml}$ ) Legionella not found. In patients with lymphocytes count less than 1500 (cell/ml), 2 (6.5\%) were positive. In ICU patients and low levels of lymphocytes, two (6.3\%) and in those with higher lymphocyte than $1500,1(2.6 \%)$ had positive urinary antigen.

\section{Discussion}

In our study, male and female were approximately infected the same, $(57.9 \%)$ men with mean age of 51.4 years. In the study of Jordi Rello and colleagues in 2001 in Spain as a retrospective in teaching hospitals, $80 \%$ of cases were men and mean age was 60 years. ${ }^{12}$ In the study of Van Manen and colleagues in the years 2005-2011 in the Dutch teaching hospitals on hospitalized community-acquired pneumonia, the average age of patients was 59 years and $84 \%$ were males. ${ }^{17}$ In the study of Poulose in 2005 in Singapore, performed on 80 patients with severe community-acquired pneumonia 55 were males and 25 females, mean age was 62 years. ${ }^{18}$ In other studies age was high and the majority was men. ${ }^{19,20}$ In the present study our patients were younger than patients in other studies, one of the reasons could be the difference in life expectancy in different countries on the other hand may be at a lower incidence of pneumonia in our region that these cases need to be investigated further. In our study a little percentage of patients $(3.6 \%)$ had positive urinary antigen. Although patients in need of ICU (4.3\%) compared to patients non in need of ICU (2.9\%) were higher, but this difference was not statistically significant. In Arancibia and colleagues research in the years 2005-2006, of 104 severe community-acquired pneumonic patients in Santiago prospectively, (8.6\%) patients had $L$. pneumophila, urinary antigen used in this study. ${ }^{21}$ Heike von baum and colleagues in 2007 in Germany in the prospective study, based on 2503 patients with community-acquired pneumonia, found that $(3.8 \%)$ of patients had Legionella, and the incidence of L. pneumophila in hospitalized patients and outpatients were similar. ${ }^{13}$ Poulose study in 2005 in Singapore prospectively was done on 80 patients with severe community-acquired pneumonia. Using urinary antigen in $2.5 \%$ of patients L. pneumophila was found. ${ }^{18}$ Our study is consistent with the studies of Van Baum and Poulose and differ Arancibia and colleagues, one of the reasons could be different weather conditions and may race issues that need to be investigated further. In our study a total of 26 patients (18.6\%) were smokers who were equal in both groups $(18.6 \%)$, although urinary antigen of Legionella in need of intensive care (15.4\%) compared to non-demanding intensive care $(0 \%)$ was higher, but this difference doesn't appear statistically significant. In Viasus and colleagues investigated retrospectively in 2013 in Spain that was conducted on patients with community-acquired pneumonia of 3934 patients, 214 (5.4\%) had L. pneumophila who were classified as having severe pneumonia $100.72 .8 \%$ of patients with $L$. pneumophila, smoking, and smoking was an independent risk factor associated with severe pneumonia, Legionella. ${ }^{20}$ Heike and colleagues in a prospective study in 2007 in Germany on patients with community-acquired pneumonia detect L. pneumophila in 94 patients, 33 patients (35\%) were smoking. ${ }^{13}$ In the study of Arancibia and colleagues on 104 severe community acquired pneumonia, 42 patients (40\%) were smoking. ${ }^{21}$ De Jager and colleagues in the years 2006-2007 in the Netherlands in a case - control study of patients with severe community-acquired pneumonia found 8 patients with $L$. pneumophila,4 patients $(50 \%)$ were smoking. ${ }^{19}$

Smoking rates in these studies were higher than our study. This may be due to: (1) lower consumption of cigarettes by our patients (2) we have not defined smoking alike. Some researchers may, for example, who have only a few times as well as cigarette smoking is considered. A total of 12 of our patients had diabetes (8.5\%), 5 patients in controls (7.1\%) and 7 patients (10\%) of cases. In diabetic patients in the control group, we had no case of Legionella ( $0 \%)$ and in no diabetics this group, 2 patients (3.1\%) were positive. In patients with diabetes who need intensive care, $(3.2 \%)$ had $L$. pneumophila but overall there was no significant relationship between diabetes and infection. In Arancibia and Cortes review; 21 (20.4\%) had diabetes. ${ }^{21}$ In reviewing Heike von Baum; a total of 62 people had diabetes, 27 cases of Legionella infection (43\%). In other words 27 out of 94 Legionella patients (35\%) had diabetes. ${ }^{13}$ In Viasus study, 41(19.2\%) of 214 patients with Legionella pneumonia, had diabetes. ${ }^{20}$ The prevalence of diabetes in our patients is lower than in other studies, the reasons could be: 1 . different life expectancy, resulting in a higher incidence of diabetes in older than in other societies, 2. Surveillance system of diabetic patients in developing countries and the development of our country not run well, 3 . The laboratory errors that is most likely to be observed in developing countries and 4. Different diets. In all of our patients; $25 \%$ had sodium less than 130 (meq/lit) that the two groups need and non-need intensive care, showed no significant difference. In no intensive care needed patients who had low sodium, 1 (6.3\%) and in those who have had sodium above $130,1(1.9 \%)$ tested positive for Legionella urinary antigen. In patients in need of intensive care lower than 130 (meq/lit) had 1 case (3.5\%); in those with higher sodium of 130 (meq/lit) however, 2 patients (3.9) were positive for Legionella urine test. Although hypernatremia in severe than non-severe Legionella was more common but statistically not significant. According to Arancibia and colleagues sodium less than 130 (meq/lit) was an independent factor predicting severe community-acquired pneumonia caused by the $L$. pneumophila. ${ }^{21}$ In study of 94 Legionella patients Heike founded, 19 patients (20\%) had sodium less than 135 (meq/lit). 29 outpatients and 65 were admitted. Hypernatremia seen in any outpatient and in $15(23 \%)$ hospitalized patients respectively. ${ }^{13}$ Viasus reviews and colleagues of those who were L. pneumophila, 173 cases of non-severe $(81 \%)$ and 41 patients $(19 \%)$ had severe pneumonia. Forty two patients with non-severe group (25.9\%) and 12 patients $(30 \%)$ in the extreme had sodium less than 130 (meq/lit) but difference in the two groups was not significant. ${ }^{20}$ The results of our survey resemble those of Viasus et al. and Heike Van Baum and Associates and differs Arancibia and colleagues. The causes of this difference can be: (1) the small number of our Legionella patients (2) difference caused by human error and gauges 3 . The difference caused by sodium intake of patients. Peripheral blood lymphocytes in the patients we see at the beginning of $1797 \pm 865$ (cell/ml) in $15 \%$ of cases, lymphopenia of less than 1000 (cell/ml), respectively. In the control group at baseline lymphocyte levels above $1500($ cell $/ \mathrm{ml}) 2$ patients $(6.5 \%)$ were Legionella urinary antigen positive. Of those need intensive care that the lymphocytes less than $1500(\mathrm{cell} / \mathrm{ml}), 2(6.3 \%)$ and normal levels of lymphocytes, $1(2.6 \%)$ were positive for Legionella urinary antigen. We had low levels of lymphocytes in $80 \%$ of Legionella patients. De Jager and colleagues in the Netherlands in 2006-2007 in reviewing the case-control study on patients who had been hospitalized community-acquired Legionella, an average of lymphocytes in initial hospitalization was low (less than 800) and introduced as a $L$. pneumophila prognostic factor. ${ }^{19}$ In this regard, more studies did not compare. 


\section{Limitations}

Our results and De jager are largely consistent. Of course, we and Mr. de Jager and Colleagues low number of patients with Legionella may not be statistically significant. Judgment seems to be more precise studies are needed in this area with a high volume of patients is Legionella.The main limitation of the current study appears to be a relatively small sample size.

\section{Conclusion}

This study was conducted to determine the presence of L.pneumophila urinary antigen and its risk factors in patients with severe pneumonia requiring hospitalization in ICU and those don't need ICU in Razi, Golestan and Emam teaching Hospital, Ahvaz, southwest of Iran, during 2015-2016. Conclusion; unlike the initial impression, in the region of study, L.pneumophila can cause respiratory infection but is an uncommon cause of pneumonia in hospitalized patients. This disease primarily involves smokers and people with immunodeficiency; especially those with low lymphocyte early in admission. Therefore, in patients with a history of smoking and severe pneumonia requiring hospitalization in ICU, Legionella should be kept in mind.

\section{Conflicts of interest}

There are no conflicts of interests among the authors.

\section{Authors' contributions}

Study concept, design and critical revision of the manuscript for important intellectual content: Sayed Mohammad Alavi, Mohammad Amin Fazeli, Sayed MojtabaMousavian, Rouhangiz Nashibi, Mohammad Javad Mohammadi; drafting of the manuscript and advisor: Mohammad Amin Fazeli and Seyed Mohammad Alavi.

\section{Funding/support}

The authors would like to thank Clinical Research Development Center, Razi, Emam and Golestan Teaching Hospitals, for the technical support and providing the facilities.

\section{Acknowledgment}

This paper is derived out from thesis of Dr Mohammad Amin Fazeli for postgraduate degree of Infectious Diseases specialist (Grant; OG -94144). The authors wish to thank the chief and personnel of Jundishapur Infectious and Tropical Research Center for supporting of this study as well as the research affair deputy of medical college for approving the study. It should know that the staff of Razi hospital, the Emam hospital pulmonary and critical care, Golestan general ward, intensive care and transplant appreciate their cooperation in this field.

\section{Appendix A. Supplementary data}

Supplementary data to this article can be found online at https:// doi.org/10.1016/j.cegh.2019.03.013.

\section{References}

1. PD G, Mandell GL, Bennett JE, Dolin R, Schwartz DA. Principles and Practice of Infectious Disease. 8 th ed. Philadelphia: Churchill Livingstone; 2015:823-846.

2. Malan AK, Martins TB, Jaskowski TD, Hill HR, Litwin CM. Comparison of two commercial enzyme-linked immunosorbent assays with an immunofluorescence assay for detection of Legionella pneumophila types 1 to 6. J Clin Microbiol. 2003;41:3060-3063.

3. Den Boer J, Yzerman E. Diagnosis of Legionella infection in Legionnaires' disease. Eur $J$ Clin Microbiol Infect Dis. 2004;23:871-878.

4. Moosavian M, Fathollahzadeh B, Amoli K, Moazzami N. Isolation of Legionella pneumophila serogroups $1,8 \& 10$ (causative agents of legionnaires' disease) from water sources in tehran, Iran. Iran Biomed J. 1998;2:83-87.

5. Todd B. Legionella Pneumonia: many cases of Legionnaire disease go unreported or unrecognized. AJN The American Journal of Nursing. 2005;105:35-38.

6. Feng C, yee L Yuvictor. Legionella Infection. Braunwald Kosper, Hausev Fauci, Jamesson Lengo. Harrison's Principles of Internal Medicine. sixteenth ed. 2005; 2005:870-874 New York.

7. Fields BS, Benson RF, Besser RE. Legionella and Legionnaires' disease: 25 years of investigation. Clin Microbiol Rev. 2002;15:506-526.

8. Alavi SM, Moshiri N, Moosavian M, Yusefi F, Abbasi E. Seroprevalence of Legionella Pneumophila in admitted patients with pneumonia in training hospitals, Ahvaz, Iran. J Med Sci. 2009:811-816.

9. Diederen BM, Peeters MF. Evaluation of Rapid U Legionella Plus Test, a new immunochromatographic assay for detection of Legionella pneumophila serogroup 1 antigen in urine. Eur J Clin Microbiol Infect Dis. 2006;25:733-735.

10. Wunderink RG, Waterer GW. Community-acquired pneumonia. $N$ Engl J Med. 2014;370:543-551.

11. Campese C, Bitar D, Jarraud S, et al. Progress in the surveillance and control of Legionella infection in France, 1998-2008. Int J Infect Dis. 2011;15:e30-e37.

12. Rello J, Bodi M, Mariscal D, et al. Microbiological testing and outcome of patients with severe community-acquired pneumonia. Chest. 2003;123:174-180.

13. von Baum H, Ewig S, Marre R, et al. Community-acquired Legionella pneumonia: new insights from the German competence network for community acquired pneumonia. Clin Infect Dis. 2008;46:1356-1364.

14. Goodarzi H, Jamaati H, Norouzi J, et al. Detecting legionella pneumophila with sputum culture and urinary antigen test in patients with acute pulmonary infection. Research in Medicine. 2005;29:351-355.

15. Kamali M, Yousefi F, Mohammadi MJ, et al. Hydatid cyst epidemiology in Khuzestan, Iran: a 15-year evaluation. Archives of Clinical Infectious Diseases. 2018;13:e13765.

16. Nashibi R, Afzalzadeh S, Mohammadi MJ, Yari AR, Yousefi F. Epidemiology and treatment outcome of mucormycosis in Khuzestan, Southwest of Iran. Archives of clinical infectious diseases. 2017;12:e37221.

17. Engel M, Van Manen L, Hoepelman A, Thijsen S, Oosterheert J. Diagnostic, therapeutic and economic consequences of a positive urinary antigen test for Legionella spp. in patients admitted with community-acquired pneumonia: a 7-year retrospective evaluation. J Clin Pathol (Lond). 2013;66:797-802.

18. Poulose V. Severe community-acquired pneumonia requiring intensive care: a study of 80 cases from Singapore. Singap Med J. 2008;49:458.

19. de Jager CP, Gemen EF, Leuvenink J, et al. Dynamics of peripheral blood lymphocyte subpopulations in the acute and subacute phase of Legionnaires' disease. PLoS One. 2013;8:e62265.

20. Viasus D, Di Yacovo S, Garcia-Vidal C, et al. Community-acquired Legionella pneumophila pneumonia: a single-center experience with 214 hospitalized sporadic cases over 15 years. Medicine. 2013;92.

21. Arancibia F, Cortes CP, Valdés M, et al. Importance of Legionella pneumophila in the etiology of severe community-acquired pneumonia in Santiago, Chile. Chest. 2014;145:290-296. 